\title{
Smart soil monitoring and water conservation using irrigation on technology
}

\author{
George Fernandez S., Vijayakumar K., Kalyanasundaram V., Vidyasagar S., Selvakumar K., \\ Selvabharathi D., Palanisamy R., Karthikeyan D. \\ Department of EEE, SRM Institute of Science and Technology, India
}

\begin{tabular}{l}
\hline \hline Article Info \\
\hline Article history: \\
Received Jul 8, 2019 \\
Revised Nov 9, 2019 \\
Accepted Jan 21, 2020
\end{tabular}

\section{Keywords:}

IoT

Standalone

User-oriented

\begin{abstract}
The conventional system of irrigation how efficient and well managed it may be wastes up to $50 \%$ of the water supplied. Purpose of this paper is to provide a user-oriented and standalone irrigation solution for soil monitoring and water conservation, based on network of sensor. Using the input from the user the system will act automatically. It not only reduce the water waste but also is cost efficient and easy to operate. Different parameters like soil moisture, temperature and humidity are imported in order to calculate the efficient quantity of water needed. This system uses different sensors like soil moisture sensor, humidity sensor and temperature sensor to take required data. In this paper, the proposed project uses sensor having low power consumption reducing load overall. The system also stores the input and uses it for forecasting. The concept of IoT is implied in this paper. The control of motors are automated powered by solar panels. The data is stored in table format. All data is accessible via internet. This system can help the user to gain vital data about the fields irrespective of the weather condition.
\end{abstract}

Copyright $@ 2020$ Institute of Advanced Engineering and Science. All rights reserved.

\section{Corresponding Author:}

Palanisamy R,

Department of EEE, SRM Institute of Science and Technology,

Chennai 603203, India.

Email: palanikrs@gmail.com

\section{INTRODUCTION}

Agriculture provides employment to more than $50 \%$ of Indian population yet it is treated inferiorly. India is touching sky in innovation and development fields, still the very need of our survival, agriculture is carried out by ancient methods. There is a reason to this slow paced development of the agricultural sector. Farmers do not tend to have complete faith on the modern day automated techniques. As most of the automated bots tend to ignore the farmers own experience and understanding of his land and agricultural practices thereby rendering unsatisfactory results. One such application in the field of agriculture is irrigation, which can be monitored by a sensor network and automated operation using a controller of a water delivery system. Despite continuous advancement, irrigation in many places of the India is a manual process driven by conventional methods because of higher costs or tough operating systems of the advanced irrigation. In Conventional System, Water is uniformly fed to the farm fields. Also, the user has to manually switch off the pump. This often results in excessive or insufficient delivery of water Irrigation plays an important role in agriculture therefore accurate and precise pumping of water is required depending upon the fields and crops sensitivity. Moreover, this leads to huge loss of electricity and water if not carefully monitored. Therefore, clean and renewable energy sources are being deployed in agricultural fields as well [1]. Digitization of the physical world is fundamentally changing industries by integrating sensor networks. So, a Sensor-based decision support and automated infrastructure control can be helpful in minimizing manual intervention [2]. In irrigation, digital data driven decisions can enable a sustainable use of water and reduce the burden of users 
too [3]. Irrigation systems that involves sensor network data with automatic control can lead to dynamic operation that adapts to the environment in real time as new data are input by the system through the sensor network therefore making embedded system based irrigation technique quite fascinating [4]. Microcontrollers take the burden of analyzing the conditions by the user, and even their mechanical effort is reduced by using relay triggered by these controllers. These microcontrollers/microprocessors collect real time sophisticated analogous data from the sensors and convert them to readable byte code and then use this data for processing the tasks, Raspberry pi is one such microprocessor which has changed the course of technology because of its compact size and amazing processing power and functionality $[5,6]$.

With the advent and growth of Internet of Things (IoT) irrigation techniques are also becoming very modernized. IoT is not just a medium of communication between the machine and the server but it also is connecting the farmer's agricultural techniques with the world [7]. The data fetched by the sensors and the kind of action taken by the processor can be monitored and controlled by the user according to his requirement thereby making the machine process a bit more flexible based on the real time data received [8]. IoT provides a medium of wireless communication over large areas thereby bringing the control over the machine on fingertips from anywhere across the world [9]. IoT communicates with the cloud thereby storing the data it receives by the processor into the database provided in the cloud memory which can be constantly viewed in real time or later through the cloud domain $[10,11]$. The IoT module can communicate with the controller in a bidirectional way or two-way communication channel, i.e. Universal Asynchronous Receiver/Transmitter (UART) channel. This two-way communication can be very helpful for the processor to take decisions based on data sent to it by the IoT module. IoT module can collect data from the client site which can be a task or an action required to be taken by the processor [12]. The data is classified depending upon the IP address for which it has been sent accordingly the IoT module sends data to the concerned machine [13-15]. There are numerous techniques of creating an automated or smart irrigation techniques using sensors and backed up by IoT to provide for communication [16-25]. But the major drawback of most of these systems are that they are completely dependent on the sensors data which might be correct to certain extent but the sensors cannot be hundred percent efficient every time moreover irrigation is a thing which is land and crop specific hence complete automation will lead to unwanted extra loss of water sometimes and may also lead to extreme consumption of energy from the grid thereby making it a very costly affair. The proposed work aims at creating a semi-autonomous irrigation system which:

a) Utilizes clean and green energy resource for operating, i.e. solar power in this case as it is easily available and is capable of supplying power to the system efficiently.

b) Uses sensors data to monitor the soil and environment to help the user to take the decision of pumping water.

c) Uses IoT to establish a source of communication between the user and the system and also displays the sensors data online.

d) Stores the sensors data and the user's response into a Sql database which can be analyzed later using the raspberry code and forecasting the action of the machine depending upon user's previous action to similar condition.

\section{PROPOSED SCHEME}

In this paper, an automated irrigation system is to be developed to optimize water usage for agricultural crops by monitoring soil. The adoption of sensors for water management in agriculture has received an increasing attention with reference to the irrigation optimization and control. The system has a distributed network of soil-moisture and temperature sensors placed in the root zone of the plants, along with rain sensors placed at various zones. All the sensor information and data is acquired and transmitted to the microcontroller. An algorithm will be developed with threshold values of temperature and soil moisture that is to be programmed into a microcontroller-based gateway to control water quantity pumped to the fields. Based on rain sensors data, control is given via an IoT module to the user to stop or start the flow of water if necessary. The actions taken by the user in the given sensor states are stored in a database created using Python, so that the system can use the user's action to make future decisions when user input is unavailable. The adoption of sensors for water management in agriculture has received an increasing attention with reference to the irrigation optimization and control. The system has a distributed network of soil-moisture and temperature sensors placed in the root zone of the plants, along with rain sensors placed at various zones. All the sensor information and data is acquired and transmitted to the microcontroller. An algorithm will be developed with threshold values of temperature and soil moisture that is to be programmed into a microcontroller-based gateway to control water quantity pumped to the fields. Based on rain sensors data, control is given via an IoT module to the user to stop or start the flow of water if necessary. The actions taken by the user in the given sensor states are stored in a database created using SQL, so that the system can use the users' action to make future decisions when 
user input is unavailable. Being an autonomous at the same time controlled method using solar power for irrigation it is very economical smart and efficient technique.

\subsection{Methodology}

In our proposed system Raspberry pi is used as a controller. A Python Script is run on the Raspberry $\mathrm{Pi}$, to code so that it reads the input parameters and proceed with the other operations. The python script will repeatedly retrieve the digital data from the MSP. It is also responsible for sending the data to IoT module, which sends the information to the user. SQLite is also used to store the database and user response by the Raspberry Pi. This database is used to forecast Raspberry response in case there is no user input. This database is also uploaded on the online server code so that it can be fetched by the user in future to check previous responses. This makes the system intelligent and precise There are three sensors connected to the Raspberry Pi. These sensors will take in different data such as temperature humidity and soil moisture. The functions and working of each sensor are explained below in this paper. In the proposed work, the sensor used for to obtain data on the soil and weather paper are soil moisture sensor used is LM393.

It has a MSP connected to it, which converts analog readings to discrete. It consists of two soil probes consist of two thin copper strips each of around $5 \mathrm{~cm}$ length which is to be immersed into the soil for soil moisture readings. The strips give a voltage output according to the conductivity of soil. The soil between the strips works as a variable resistance whose value depends upon moisture content material in soil. This variable resistance helps in calculating the readings in terms of analog voltage. This analog output is converted to digital form by the MSP. Humidity sensor is used in calculating the water content in the atmosphere. There are various reasons for which the humidity sensor can be helpful for this paper. Humidity in air also helps in predicting the rain sometimes. Mainly there are three types of humidity sensors: Capacitive, Resistive and Thermal. They detect the change in conductivity in the atmosphere and find the correspondence humidity. The temperature sensor used for this paper is LM35. It provides a precise centigrade temperature. Since the water soil of any type of soil depends on temperature too, it is necessary to find the temperature. The Pin diagram of the sensor is shown in Figure 1. The MCP model used for this paper is MCP3008. It is an 8-channel 10bit analog to digital converter (ADC) which uses the SPI bus protocol and obtains analog inputs for the Raspberry Pi. It has 8 analog inputs and it engages only four pins of Raspberry Pi except the power and ground pins. The following is the pin diagram of the MCP. $\mathrm{CH} 0-\mathrm{CH}-7$ are the input pins for the analog signal, whereas the PIN 9-16 have different functions. D Out and $\mathrm{D}_{\mathrm{IN}}$ are the two pins connected to the Raspberry Pi, used for sending and receiving data. CLK is used for giving the clock signal. The input power is obtained from Solar Energy. The Power supply is the general solar power circuit which consists:

a) Solar Panel

b) DC-DC Converter

c) Battery

An Auxiliary power source from grid is also kept as backup in case of bad weather conditions or if the solar power is not sufficient enough to run the system. An IoT module is a little electronic gadget installed in items, machines and things that interface with remote systems and sends and gets information. Some of the time alluded to as a "radio chip", the IoT module contains a similar innovation and information circuits found in cell phones however without highlights like a presentation or keypad. Another key differentiator of IoT modules is that they give dependably on network. This is on the grounds that IoT applications need to send information naturally, continuously without somebody hitting a send catch. Furthermore, they are designed for outrageous sturdiness and life span and need to work constantly for 10 years or more to legitimize the business case and cost of the innovation. It communicates with the Raspberry pi through the UART channel in a two-way medium. Python is an exceptionally helpful programming language that has a simple to peruse sentence structure, and enables developers to utilize less lines of code than would be conceivable in dialects, for example, get together, C, or Java. The Python programming language really began as a scripting language for Linux. Python programs are like shell contents in that the documents contain a progression of directions that the PC executes start to finish. This is the default language that accompanies the Raspberrian OS. SQLite is a social database the board framework contained in a $\mathrm{C}$ programming library. Rather than numerous others database the board frameworks, SQLite isn't a client-server database motor. Or maybe, it is inserted into the end program. SQLite is an inserted SQL database motor. Dissimilar to most other SQL databases, SQLite does not have a different server process. SQLite peruses and composes legitimately to conventional circle records. A total SQL database with numerous tables, lists, triggers, and perspectives, is contained in a solitary circle record. The database record design is cross-stage - you can openly duplicate a database between 32-bit and 64-bit frameworks or between enormous endian and little-endian structures. These highlights settle on SQLite a well-known decision as an Application File Format. SQLite database documents are a prescribed stockpiling design by the US Library of Congress. Consider SQLite not as a trade for Oracle but rather as a substitution for fopen (). Relay is an electromagnetic switch worked by a moderately little electric flow that 
can switch on or off a lot bigger electric flow. The core of a transfer is an electromagnet (a loop of wire that turns into an impermanent magnet when power courses through it). It is a kind of electrical lever: switch it on with a modest flow of current and it switches on ("influences") another apparatus utilizing a lot greater current. The water pumping system is solar powered. It is connected with a relay which acts as a switch between the Raspberry Pi and the motor. When the relay is triggered by the Raspberry Pi, It commands the motor to run. The power circuit is used to charge the dc source battery which is used to power the Raspberry pi module along with the sensors networks and the water pump. The power circuit comprises of solar panel and dc-dc converter, the panel rating depends upon the number of components connected to the raspberry pi.

\subsection{Operational strategy}

Once the pi is powered up the sensors get activated and start accumulating data from the soil contents and weather. The sensors namely soil moisture, humidity and temperature sensors (Figure 2), which are analog sensors are constantly receiving the values from the surrounding environment. A delay is introduced in the process of reading sensor values so as to minimize the consumption of memory for data storage and moreover a delayed reading would give a more approximate value rather than constantly fluctuating data. This data is converted from analog to digital using MCP3008 which is an analog to digital converter based on successive approximation technique; it converts the analog voltage values received from the sensors to its digital counterparts so that it can be interpreted by the Raspberry pi. Raspberry pi having a good processing speed of about $1.2 \mathrm{GHz}$ and RAM of about $1 \mathrm{~GB}$ suits perfectly for our application as we can continuously communicate with the sensors with SPI protocol and IoT module using UART channel continuously. The sensor data after being processed by the ADC is received by the Raspberry Pi. The Raspberry pi has been loaded with the Raspberrian OS to provide a GUI and operating platform for the operation and control of the microprocessor. Using the console, the required files are installed i.e. Python which is by default present is upgraded, MYSQL for database creation, IoT module interface etc. Once the files are updated and ready to operate.

In this work Python is used for coding the Raspberry Pi, as it is Object Oriented, easy to code and available in the Raspberrian OS by default. The Pi is coded so as to receive all the data from the ADC and it transfers these data to the server by communicating via the IoT module. Further depending on the soil conditions and his experience the user will decide whether the conditions are suitable for pumping water or not. This decision of the user is first recorded at the client side then sent to the server side and from there it is transferred back to the Raspberry Pi via the IoT module depending on the I.p. address mentioned, as it is a two-way communication channel. The data from the sensors and the user's action in its response is stored into the SQL database, when the user input is unavailable for more than half an hour the present sensor conditions are compared with the data stored in the database and whichever value is found to be near to the current data the action corresponding to that situation is carried out. This is done by retrieving the values from the database and storing each value in a unique object, finally these objects are stored into an array of objects. Finally, the current object variables are compared with the previous objects by iterating through the array. Whenever the value is found to be close to any previous objects value it gives the action corresponding to that state. Thereby making the system semi-autonomous. the table will contain the data for the given parameters. The data will be taken in from the sensors after a given interval of time. Each time the user will receive information regarding the data collected and will be asked for an input whether or not to release the water to the fields. If the user responds in favour of letting the water in the fields then it will be done so and if not then there will be no work done by the motors. In case the user is unable to send a response within a given interval of time, the Raspberry Pi will use machine learning algorithm to find previous condition similar to the present one and will act according to the action proposed by the user that time. If the Raspberry Pi is unable to find a suitable condition it will ask the user for another response and will continue to wait for it. The sample table of the database and its working as shown in Tables 1 and 2.

In the Tables 1 and 2 sets of databases, the Raspberry Pi doesn't receive any response from the user whether to run the motor or not. So, it compares the obtained readings from the previous inputs from the sensors to the corresponding user input. It tries to find the similar sensor input from the previous data and uses it corresponding user input as the user input for the current conditions.

Table 1. Automatic action undertaken when no user input was found for a specified time

\begin{tabular}{ccccc}
\hline Time Interval & Temperature & Humidity & Soil moisture & User input \\
\hline 1 & 30.2 & $39 \%$ & $37.4 \%$ & no \\
2 & 33.5 & $40 \%$ & $35.2 \%$ & yes \\
3 & 31.1 & $37 \%$ & $40 \%$ & no \\
4 & 30.4 & $39 \%$ & $38.2 \%$ & no \\
5 & 29.8 & $39.4 \%$ & $39.4 \%$ & no \\
\hline
\end{tabular}

Indonesian J Elec Eng \& Comp Sci, Vol. 19, No. 1, July 2020 : 99 - 107 
Table 2. Analyzed data

\begin{tabular}{ccccc}
\hline Time Interval & Temperature & Humidity & Soil moisture & User input \\
\hline 1 & 30.2 & $39 \%$ & $37.4 \%$ & no \\
2 & 33.5 & $40 \%$ & $35.2 \%$ & yes \\
3 & 31.1 & $37 \%$ & $40 \%$ & no \\
4 & 30.4 & $39 \%$ & $38.2 \%$ & no \\
5 & 29.8 & $39.4 \%$ & $39.4 \%$ & no \\
6 & 30.1 & $38.1 \%$ & $35.4 \%$ & -(yes) \\
\hline
\end{tabular}

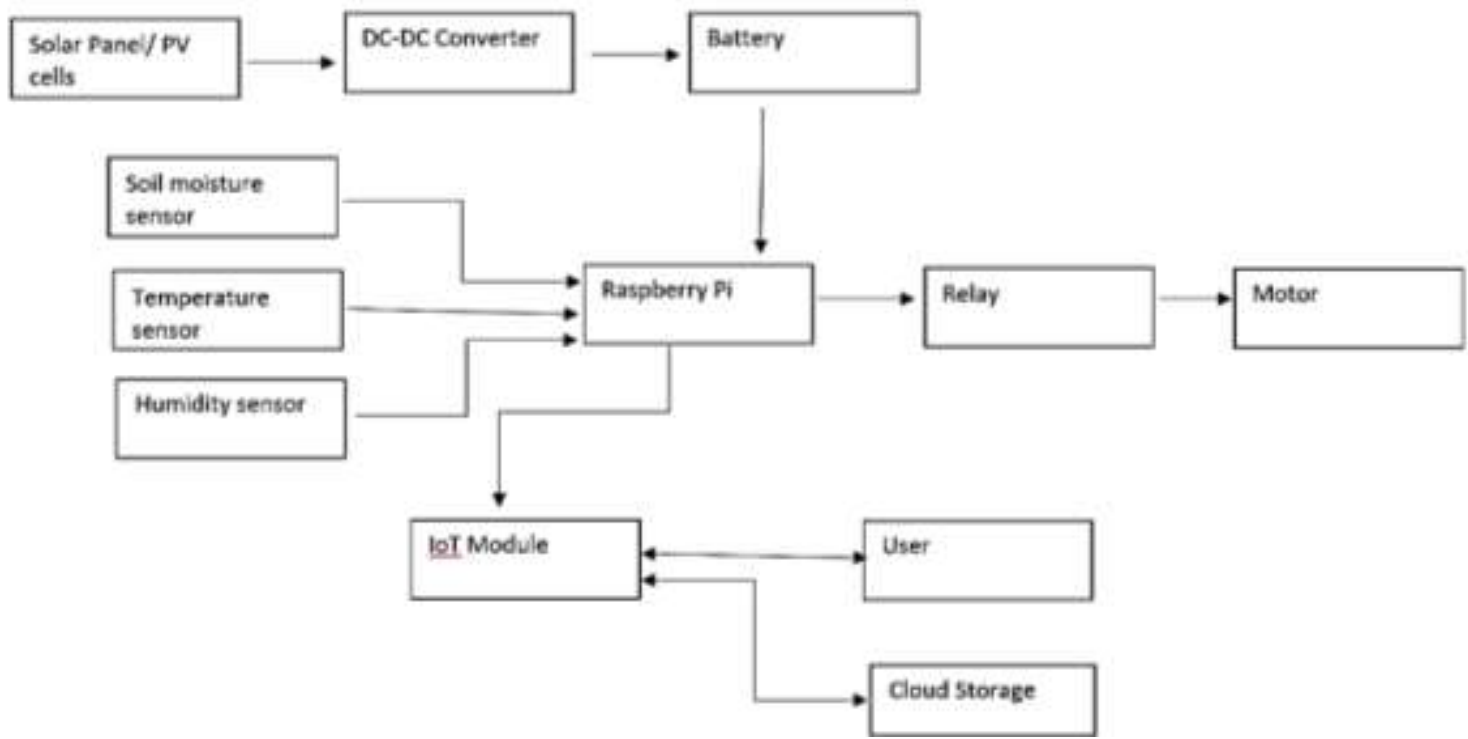

Figure 1. The block diagram for the paper

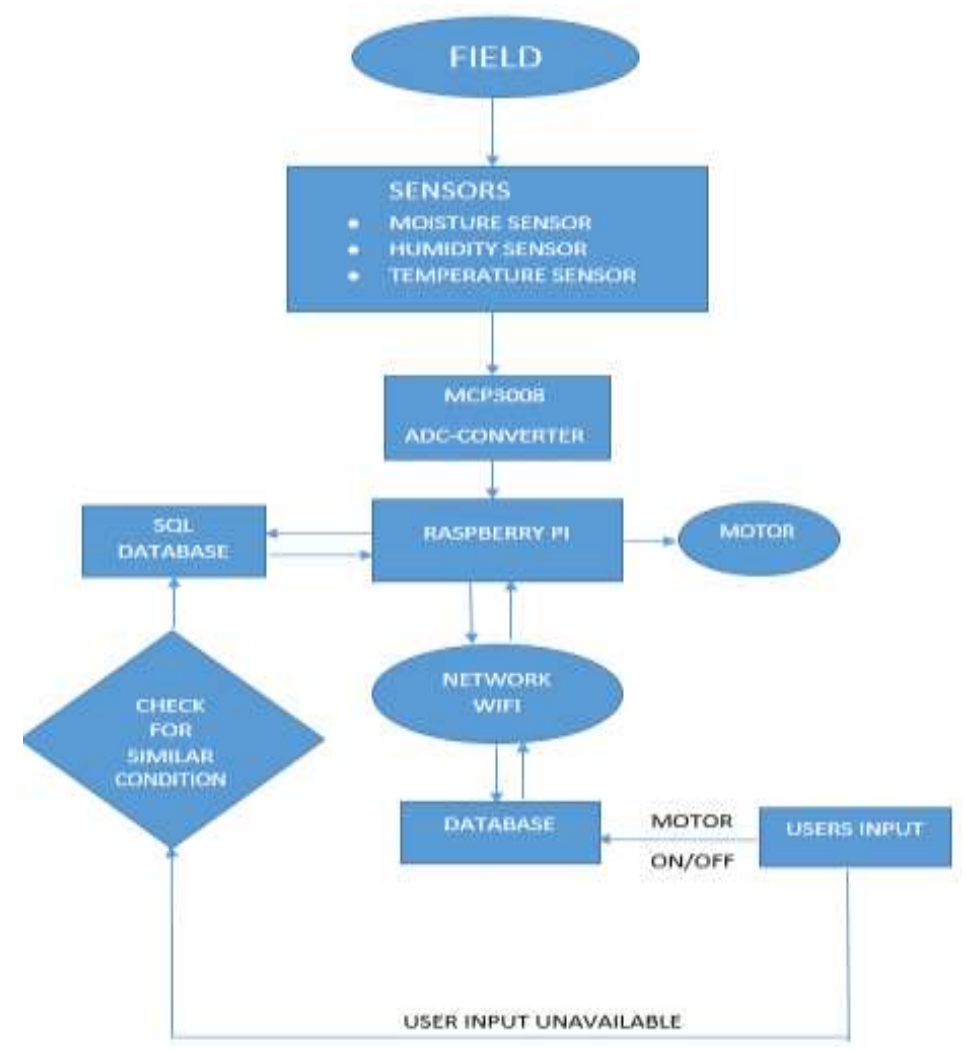

Figure 2. Flow chart 


\section{RESULTS AND DISCUSSION}

The system is tested for in a prototype container containing soil and a flower plant. The sensors are continuously monitoring the soil conditions and environment for the crop. The users input are also constantly fed to the portal for different conditions these actions get stored in the database after feeding some values the system is tested to run under the similar conditions but without the users input this time. The system autonomously follows the previous actions fed by the user to the system. The login page allows the user to have security and access is not open for public. Different login pages can be setup for different iots accordingly so that if the farmer uses multiple systems can keep track of all using a single site. This increases the security of our system and helps the farmer in management of wide area crops. The test on a prototype as shown in Figure 3-6.

Log 1 indicates the temperature in ${ }^{\circ} \mathrm{C}$, soil moisture and humidity. It is being stored in the cloud for future reference. This will help us to use the data later on for forecasting purposes. The data is easily accessible using the internet. Farmers can check at what time of the day water level was high or low and accordingly can set the pump on or off. No of devices to be connected can vary and be controlled via the Internet itself. Here the hardware prototype is shown as assembled. There a raspberry pie's gpiu is set to board mode. All the sensors are connected to MCP3008 and it is connected to the raspberry pie. It is the ADC required to convert the analog signal coming from the sensors to digital signal. This digital signal is sent to the raspberry pie using serial communication interface. This data once received is then sent to the IoT module connected. This uses UART communication and is stored in the cloud. This Iot module also helps us to receive the signal from the user to start or stop the motor. This model will be useful for the farmers to keep a check on their crop land via IoT and will enable them to take on spot decisions regarding their plans for their crops. This will reduce the burden on the farmers to regularly go on to their fields during bad weather conditions. This will also enable the farmer to control over or under irrigation of their land. This will help the farmers to control their water supply more fruitfully and will reduce wastage. The time will be cut short using this method as compared to the conventional ones. The power supply is renewable so it reduces the load on the grid. Water conservation is high as compared to previous methods as here users control over the system maintains an order.

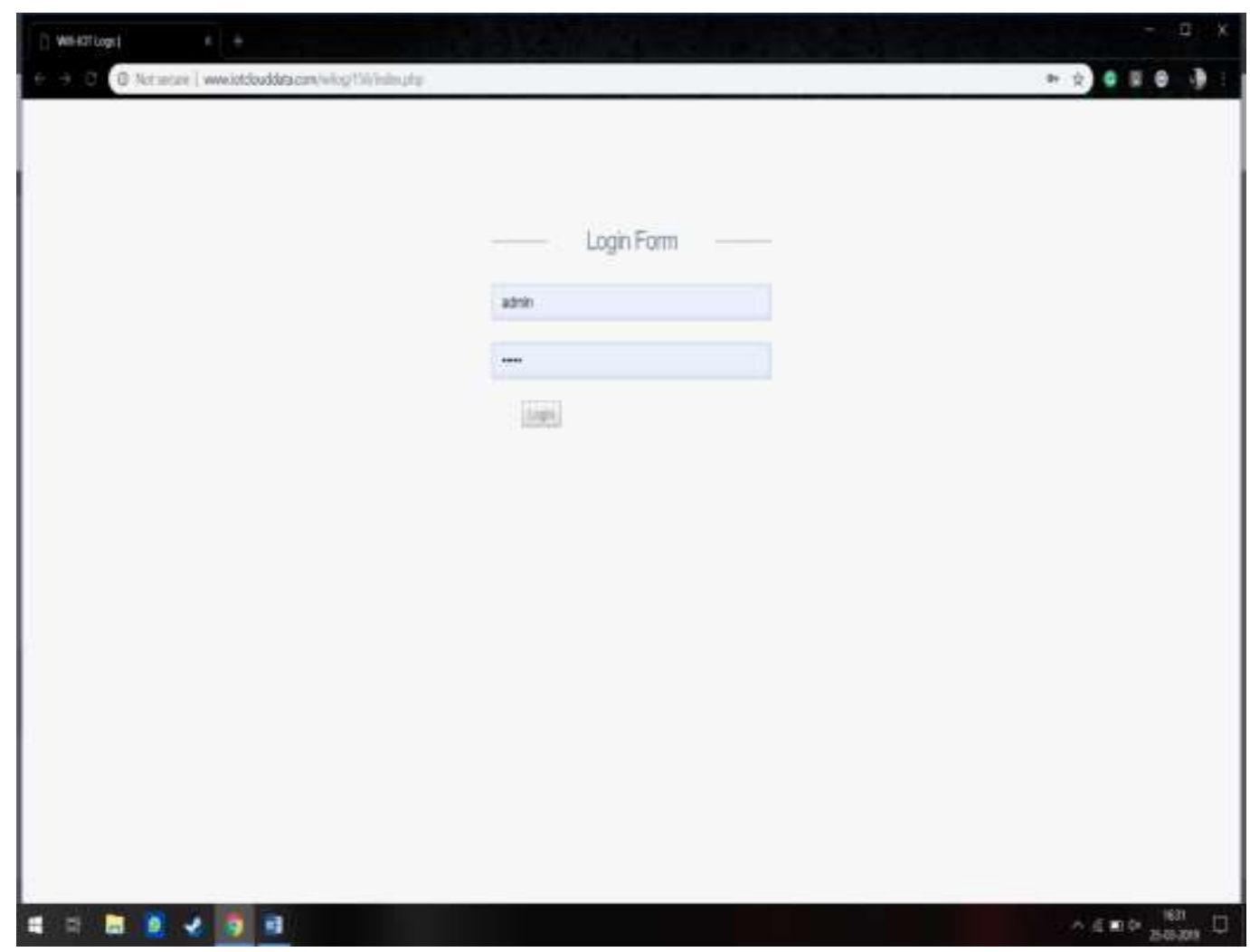

Figure 3. Home page 


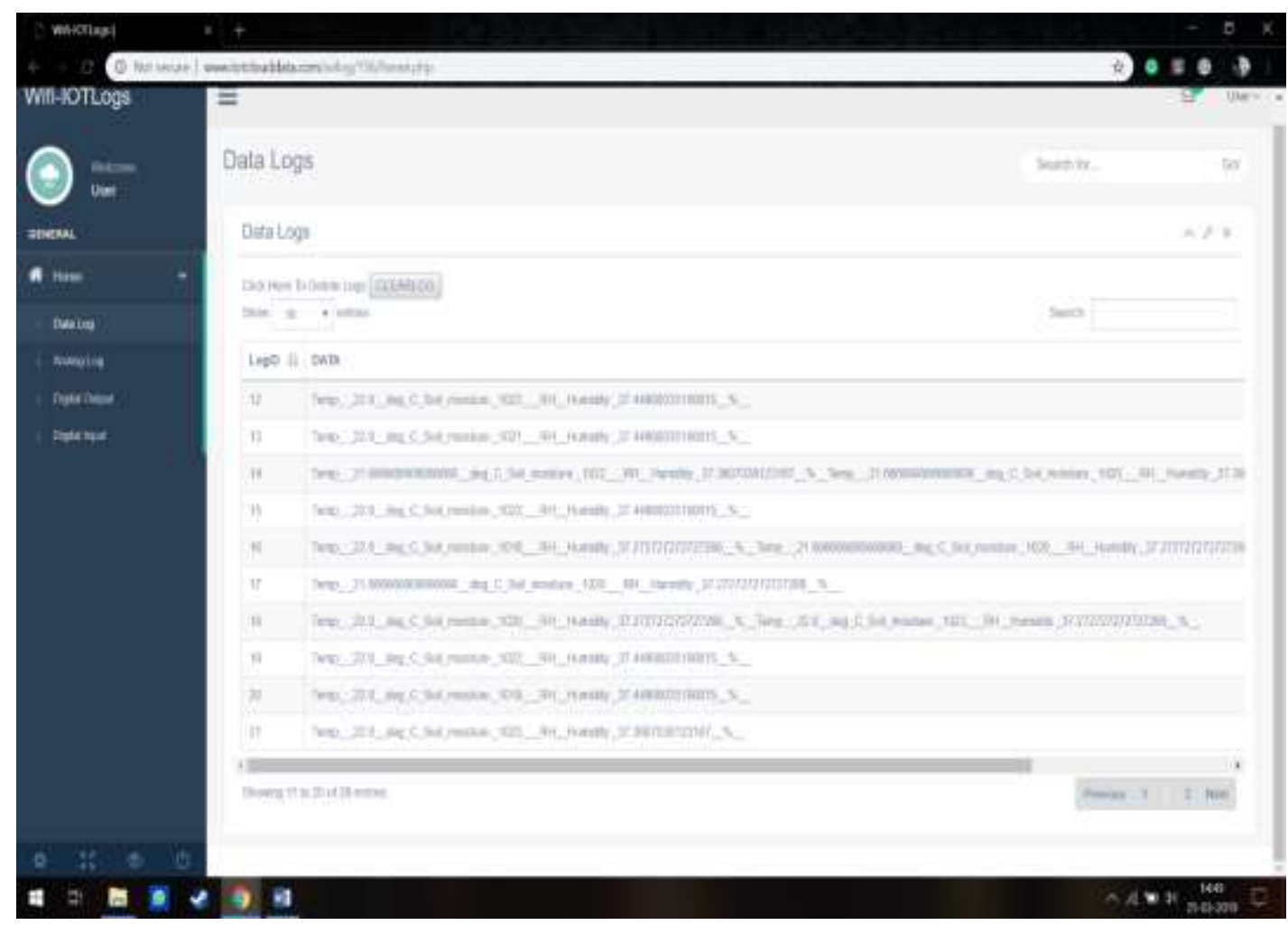

Figure 4. Data being logged

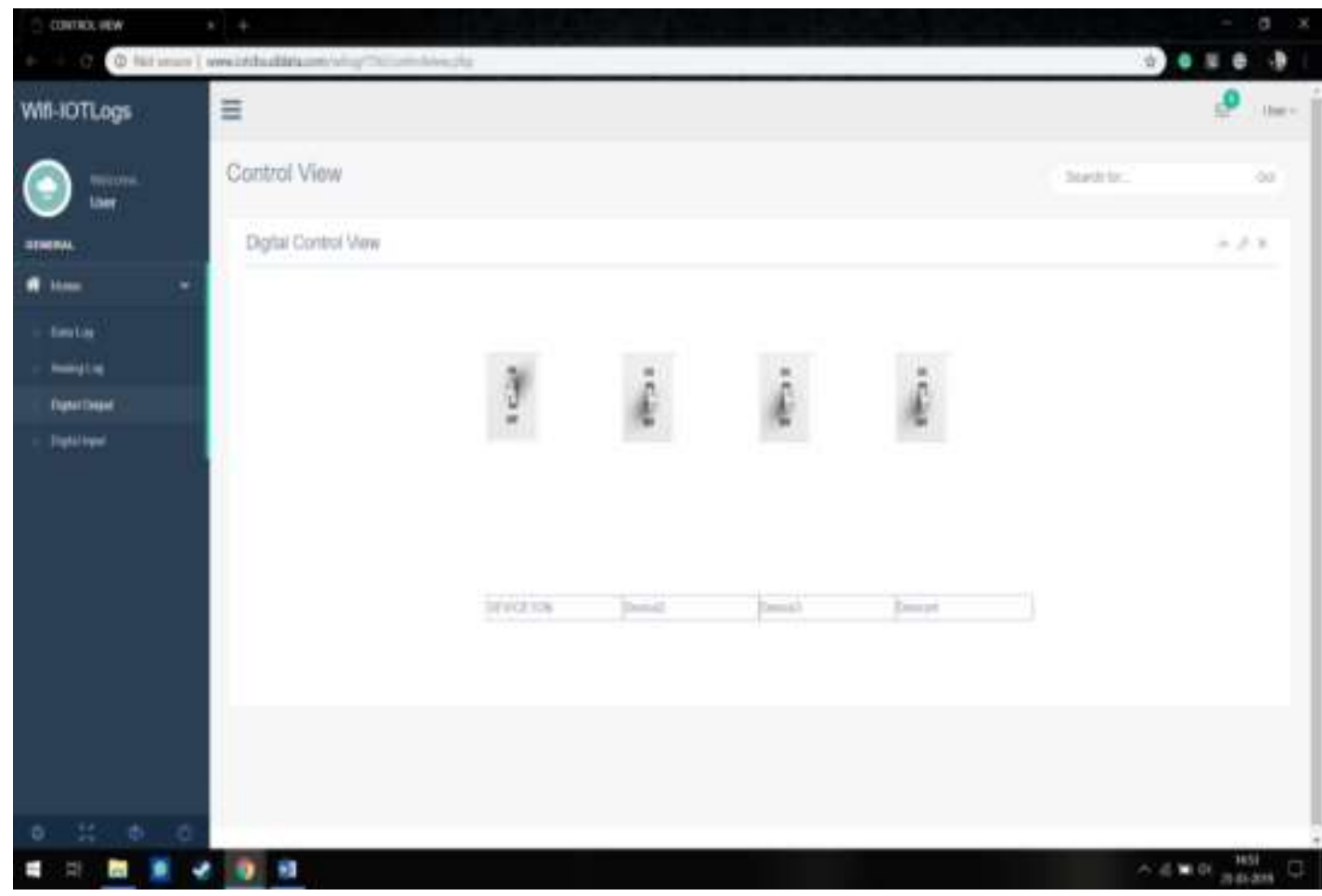

Figure 5. The digital control 


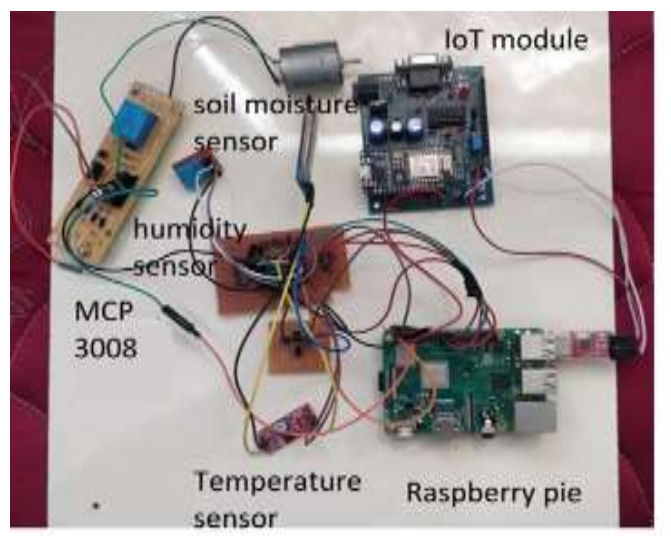

Figure 6. Hardware prototype assembly

\section{CONCLUSION}

Conserving water and electricity in the agricultural sector is the need of the hour. In this paper a sensor based technique for analyzing the conditions for irrigation, makes the process more controllable by rendering real time data to the processor making the process reliable as it is less manual. The sensor data was successfully transferred to the cloud storage to be viewed online and the user data could be accepted. The user action along with the sensor data is fed to the Sql database and further when user input is unavailable the processor is made to follow the pattern suggested by the user through his previous inputs for similar conditions.

\section{REFERENCES}

[1] Junyong Liu, Member, IEEE, Yanxin Chai, Yue Xiang, Member, IEEE, Xin Zhang, Member, IEEE, Si Gou, Member, IEEE, and Youbo Liu, Member, IEEE, "Clean Energy Consumption of Power Systems Towards Smart Agriculture: Roadmap, Bottlenecks and Technologies ”CSEE Journals \& Magazines, vol 4, issue 3, 2018.

[2] Rahim khan ihsan ali, muhammad zakarya, mushtaq ahmad, muhammad imran, and muhammad Shoaib, "Technology-Assisted Decision Support System for Efficient Water Utilization: A Real-Time Testbed for Irrigation Using Wireless Sensor Networks ",10.1109/ACCESS.2018.2836185, IEEE Access, dt of publishing 14.03.2018

[3] Joaquín Gutiérrez, Juan Francisco Villa-Medina, Alejandra Nieto-Garibay, and Miguel Ángel Porta-Gándara "Automated Irrigation System Using a Wireless Sensor Network and gprs module," IEEE Transactions On Instrumentation And Measurement, vol. 63, no. 1, january 2014.

[4] Sabrine khriji, dhouha e houssaini, mohamed wassim jmal, christian viehweger, mohamed abid, olfa kanoun "Precision irrigation based on wireless sensor network, " IET Science, Measurement \& Technology, Volume: 8 , Issue: 3 ,May 2014.

[5] Chris Edwards, Engineering \& Technology, "Not-So-Humble Raspberry Pi Gets Big Ideas”,Vol 8,Issue 3, April 2013.

[6] F.C. Lee; P. Barbosa; Peng Xu ; Jindong Zhang ; Bo Yang ; F. Canales, "Topologies And Design Considerations For Distributed Power System Applications", Proceedings Of The IEEE ( Volume: 89, Issue: 6, Jun 2001.

[7] Nurzaman Ahmed, Debashis De, Senior Member, Ieee, And Md. Hussain, Member, IEEE, "Internet of Things (Iot) For Smart Precision Agriculture and Farming In Rural Areas”, IEEE Internet Of Things Journal, Vol. 5, No. 6, December 2018

[8] Savari, G.F.; Krishnasamy, V.; Sathik, J.; Ali, Z.M.; Aleem, S.H.A. Internet of Things based real-time electric vehicle load forecasting and charging station recommendation. ISA Trans. 2019.

[9] Rahul D S, Sudarshan S K, Meghana K, Nandan K N, R Kirthana and Pallaviram Sure Electronic and Communication Engineering, Faculty of Engineering and Technology M. S. Ramaiah University of Applied Sciences Bangalore, "iot based Solar Powered Agribot for Irrigation and Farm Monitoring", India proceedings of the Second International Conference on Inventive Systems and Control (ICISC 2018).

[10] Geng Yang; Mingzhe Jiang; Wei Ouyang; Guangchao Ji; haiboxie; Amir M. Rahmani; pasililjeberg; hannutenhunen, "iot-Based Remote Pain Monitoring System: From Device to Cloud Platform" IEEE Journal of Biomedical and Health informaticsyear: Volume: 22, Issue: 6, 2018.

[11] Brunno Vanelli; Madalena Pereira da Silva; guilhermemanerichi "Internet of Things Data Storage Infrastructure in the Cloud Using nosql Databases", ; Alex Sandro Roschildt Pinto IEEE Latin America Transactions, Volume: 15, Issue: 4 , April 2017.

[12] George Fernandez.S, K. Vijayakumar and R. Palanisamy, "GPS \& GSM Based Accident Detection and Auto Intimation," Indonesian Journal of Electrical Engineering and Computer Science (IJEECS), vol. 11(1), 2018. 
[13] Chandra S. Bontu., Shaliniperiyalwar., Mark Pecen,"Wireless Wide-Area Networks for Internet of Things: An Air Interface Protocol for iot and a Simultaneous Access Channel for Uplink iot Communication” IEEE Vehicular Technology Magazine, Vol 9, Issue: 1, March 2014.

[14] Savari GF, Krishnasamy V, Guerrero JM. Optimal scheduling and economic analysis of hybrid electric vehicles in a microgrid. Int J Emerging Electric Power Syst. Vol 19, issue 6, 2018.

[15] S. George Fernandez, K. Vijayakumar, R Palanisamy," Unmanned and autonomous ground vehicle" International Journal of Electrical and Computer Engineering, Vol 9, No 5,2019.

[16] P.A. Patil, B.G. Jagyasi, J. Raval, N. Warke, P.P. Vaidya, "Design and development of wearable sensor textile for precision agriculture", 7th International Conference on Communication Systems and Networks (COMSNETS), pp. 1-6, 6-10 Jan. 2015.

[17] L.M. Rodrigues, G. Pereira Dimuro, D. Teixeira Franco, J.C. Fachinello, "A system based on interval fuzzy approach to predict the appearance of pests in agriculture", Joint IFSA World Congress and NAFIPS Annual Meeting (IFSA/NAFIPS), pp. 1262-1267, June 2013.

[18] K. Kanda, T. Ishii, T. Kameoka, K. Saitoh, R. Sugano, "Field monitoring system using Agri-Server", Proceedings of SICE Annual Conference (SICE), pp. 2069-2072, 13-18 Sept. 2011.

[19] J.B. Sanger, H. Sukoco, S.K. Saptomo, "Reliable data delivery mechanism on irrigation monitoring system", International Conference on Advanced Computer Science and Information Systems (ICACSIS) 2014, pp. 53-56, 18-19 Oct. 2014.

[20] Adriansyah, A.W. Dani, "Design of Small Smart Home system based on Arduino", Electrical Power Electronics Communications Controls and Informatics Seminar (EECCIS) 2014, pp. 121-125, Aug. 2014.

[21] S.R.R. Dhiwaakar Purusothaman, R. Rajesh, V. Vijayaraghavan, K.K. Bajaj, "Design of Arduino-based communication agent for rural Indian microgrids", IEEE Innovative Smart Grid Technologies - Asia (ISGT Asia) 2014, pp. 630-634, May 2014.

[22] J.M. Gomes, P.M. Ferreira, A.E. Ruano, "Implementation of an intelligent sensor for measurement and prediction of solar radiation and atmospheric temperature", IEEE 7th International Symposium on Intelligent Signal Processing (WISP) 2011, pp. 1-6, 19-21 Sept. 2011.

[23] M.S. Perez, E. Carrera, "Time synchronization in Arduino-based wireless sensor networks", Latin America Transactions IEEE (Revista IEEE America Latina), vol. 13, no. 2, pp. 455-461, Feb. 2015.

[24] S. George Fernandez, K. Vijayakumar," A Fully Automated Lawn Mower Using Solar Panel “Jour of Adv Research in Dynamical \& Control Systems, Vol. 10, 07-Special Issue, 2018.

[25] V. C. Bojan, I. G. Raducu, F. Pop, M. Mocanu, V. Cristea, "Cloud-based service for time series analysis and visualisation in Farm Management System", IEEE International Conference on Intelligent Computer Communication and Processing (ICCP), pp. 425-432, 2015. 